05,04

\title{
Асимметричность колебаний доменных границ в гармоническом и импульсном магнитных полях в кристаллах ферритов-гранатов с дрейфом полосовой доменной структуры
}

\author{
(С) Л.А. Памятных, Д.С. Мехоношин, С.Е. Памятных, Л.Ю. Агафонов, М.С. Лысов, Г.А. Шматов
}

Уральский федеральный университет им. Б.Н. Ельцина,

Екатеринбург, Россия

E-mail: Lidia.Pamyatnykh@urfu.ru

(Поступила в Редакцию 11 октября 2018 г.)

\begin{abstract}
В кристаллах ферритов-гранатов с разным характером магнитной анизотропии в переменном гармоническом магнитном поле обнаружено асимметричное смещение доменных границ при их колебательном движении и асинхронность изменения скоростей соседних доменных границ при амплитудах поля, меньших поля начала дрейфового движения доменной структуры. Установлено, что в биполярном импульсном магнитном поле смещение доменных границ в направлении дрейфа превосходит величину смещения доменных границ в противоположном дрейфу направлении. Предложена теоретическая модель, в которой асимметричность колебаний доменных границ и дрейф возникают вследствие анизотропии параметра затухания.
\end{abstract}

Работа выполнена в рамках базовой части госзадания Министерства образования и науки РФ (проекты № 3.6121.2017, 3.5719.2017).

DOI: 10.21883/FTT.2019.03.47240.280

\section{1. Введение}

В последнее время процессы, связанные с динамикой магнитной доменной структуры (ДС) и движением доменных границ (ДГ) в переменных магнитных полях, привлекают большое внимание исследователей. Одной из причин такого внимания является перспектива создания нового типа запоминающих устройств, основанных на движении доменных границ [1]. Ключевым эффектом, необходимым для создания таких устройств, является движение массива ДГ в одном направлении в ответ на определенное воздействие. Достичь данного эффекта можно с помощью приложения периодического магнитного поля (гармонического или импульсного). Например, в [2] однонаправленное движение ДГ достигалось в результате действия переменного поля на ДГ, помещенную в искусственно созданный асимметричный потенциальный рельеф. В [3] было продемонстрировано направленное движение цилиндрических магнитных доменов (ЦМД) под действием переменного магнитного поля в многослойных металлических пленках с хиральными ДГ, обладающими анизотропией скорости движения относительно направления магнитного поля.

Поступательное движение ДГ под действием осциллирующего магнитного поля известно как дрейф доменных границ. Экспериментально дрейф впервые был обнаружен в [4] в монокристаллах кремнистого железа, позже наблюдался в ферримагнетиках - пластинах ферритовгранатов [5], в слабом ферромагнетике $\mathrm{DyFeO}_{3}$ [6]. Имеется ряд теоретических работ, показывающих возможность дрейфа ДГ в переменных полях и раскрывающих возможные механизмы этого движения [7-9]. В качестве причины дрейфа указывают различие плотности энергии по разные стороны от ДГ, что приводит к возникновению эффективного давления на ДГ [10], либо различную подвижность ДГ при движении в противоположных направлениях [11]. Ряд авторов связывают возникновение дрейфа с наличием различных неоднородностей в образце, указывается, что к дрейфу ДГ может приводить различие скоростей доменных границ на краях образца [12], зарождение новых доменов на краю образца или в области градиентного магнитного поля $[13,6]$, зарождение и аннигиляция магнитных дислокаций дефектов полосовой ДС [14,15].

Для возникновения дрейфового движения необходимо нарушение симметрии при движении ДГ в противоположных направлениях. Можно полагать, что такие асимметричные эффекты проявляются не только при дрейфе ДГ, но и при колебаниях ДГ в переменных полях. Цель настоящей работы заключалась в установлении связи особенностей колебаний ДГ с дрейфом доменной структуры в гармоническом и периодически повторяющемся импульсном магнитных полях. Исследования проведены на монокристаллических пластинах ферритовгранатов со сложным характером магнитной анизотропии. Экспериментальные результаты сопоставляются с результатами численного моделирования движения ДГ, проведенного в предположении анизотропии параметра затухания.

\section{2. Образцы и методика эксперимента}

Сообщаются результаты исследований колебаний и дрейфа доменных границ в пластинах-(111) монокри- 
Параметры исследованных образцов

\begin{tabular}{c|c|c|c|c|c|c|c|c|c}
\hline № & Состав & Ориентация & $\begin{array}{c}L \\
\mu \mathrm{m}\end{array}$ & $\begin{array}{c}M_{s} \\
\mathrm{G}\end{array}$ & $\begin{array}{c}K_{1} \\
\mathrm{erg} / \mathrm{cm}^{3}\end{array}$ & $\begin{array}{c}K_{u} \\
\mathrm{erg} / \mathrm{cm}^{3}\end{array}$ & $\begin{array}{c}K_{p} \\
\mathrm{erg} / \mathrm{cm}^{3}\end{array}$ & $\left|K_{1}\right| / K_{u}$ & $K_{p} / K_{u}$ \\
\hline 1 & $(\mathrm{TbErGd})_{3}(\mathrm{FeAl})_{5} \mathrm{O}_{12}$ & $(111)$ & 73 & 40 & $-3.4 \cdot 10^{3}$ & $5.5 \cdot 10^{3}$ & $3.2 \cdot 10^{3}$ & 0.62 & 0.58 \\
2 & $(\mathrm{TbErGd})_{3} \mathrm{Fe}_{5} \mathrm{O}_{12}$ & $(111)$ & 50 & 19 & $-6.5 \cdot 10^{3}$ & $0.9 \cdot 10^{3}$ & $12.7 \cdot 10^{3}$ & 7.2 & 14
\end{tabular}

Примечание. $L-$ толщина пластины, $M_{s}-$ намагниченность насыщения, $K_{1}-$ константа магнитокристаллической анизотропии, $K_{u}, K_{p}$ - константы наведенной одноосной и ромбической анизотропии соответственно.

сталлов редкоземельных ферритов-гранатов в магнитном поле $H=H_{0} \sin (2 \pi f t)$, приложенном перпендикулярно поверхности образцов. Амплитуда магнитного поля варьировалась от 0 до $300 \mathrm{Oe}$, а частота - в интервале 10-1200 Нz. Параметры образцов приведены в таблице, а на рис. 1, $a, b-$ изображения ДС в размагниченном состоянии. Образцы имеют сложный характер магнитной анизотропии: в них присутствует магнитокристаллическая кубическая анизотропия с константой $K_{1}$, наведенная одноосная анизотропия, которая характеризуется константой $K_{u}$, и ромбическая анизотропия с константой $K_{p}$. В первом образце $K_{u}>K_{1}$, во втором образце $K_{1}>K_{u}$. Значение константы ромбической анизотропии в первом образце в 4 раза меньше значения $K_{p}$ второго образца. Модели ДС пластин приведены на рис. $1, c, d$. Далее образцы 1 и 2 будут обозначаться как одноосный и многоосный соответственно.

Доменная структура визуализировалась с помощью магнитооптического эффекта Фарадея и фиксировалась на видеокамеру, для подсветки использовался твердо-

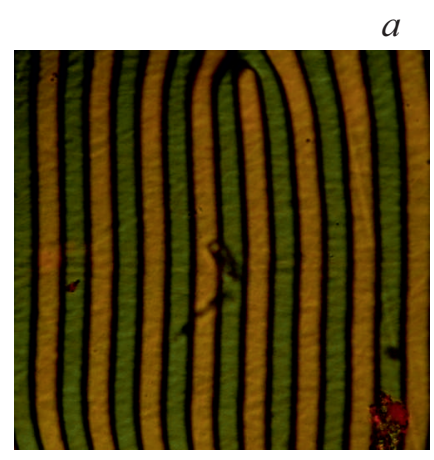

$50 \mu \mathrm{m}$
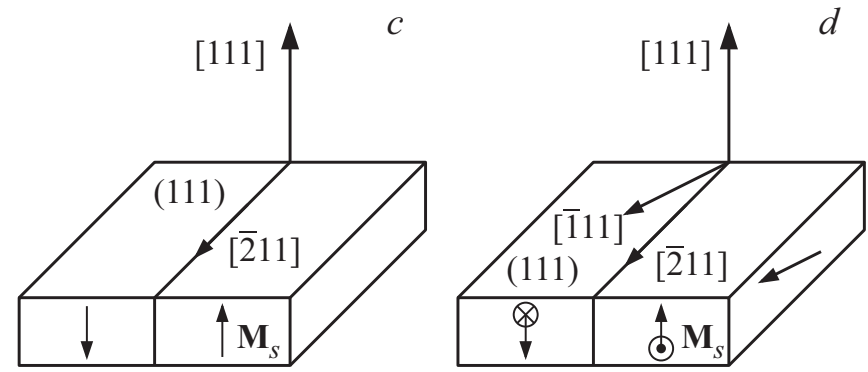

Рис. 1. Изображения доменной структуры пластин ферритовгранатов в размагниченном состоянии: $a-$ одноосный, $b-$ многоосный образец. Модели доменных структур $c-$ одноосного, $d-$ многоосного образца. тельный импульсный лазер с длиной волны $527 \mathrm{~nm}$ и средней продолжительностью импульса $30 \mathrm{~ns}$. Малая по сравнению с периодом магнитного поля $(T=1-100 \mathrm{~ms})$ продолжительность импульса лазера позволила фиксировать на каждом кадре видеозаписи мгновенное изображение динамической ДС образца. Для изучения динамики ДС была реализована стробоскопическая методика: частота переменного магнитного поля выбиралась кратной скорости съемки видеокамеры (25 fps), а частота следования импульсов подсветки устанавливалась равной скорости съемки. При этом генерация импульсов подсветки осуществлялась в моменты времени, соответствующие определенной (выбранной) фазе гармонического поля. При исследовании колебаний ДГ (в отсутствие дрейфа) фаза периодически изменялась с шагом в $1 \mathrm{deg}$, и на полученных магнитооптических изображениях при помощи компьютерной обработки автоматически определялись положения ДГ, что позволяло отслеживать их движение со временем в течение периода поля. При исследовании дрейфа ДС фиксировалась фаза поля, в которую осуществлялась подсветка, а специально разработанное программное обеспечение позволяло определять скорость движения ДГ. Форма и фаза создаваемого магнитного поля контролировались с помощью датчика Холла.

Для одноосного образца исследование колебаний и дрейфа доменных границ в периодических импульсных магнитных полях выполнено при длительности импульсов $\tau=0.24-5.0 \mathrm{~ms}$ с длительностью фронтов $\tau_{f}$ от 0.16 до $2.0 \mathrm{~ms}$. Импульсный сигнал создавался программно, подавался на аудио усилитель мощности и далее на 150 -витковую катушку диаметром $25 \mathrm{~mm}$, что позволяло формировать импульсы поля амплитудой до $300 \mathrm{Oe}$.

\section{3. Дрейф и колебания доменных границ в гармоническом магнитном поле}

В гармоническом магнитном поле, приложенном перпендикулярно плоскости образца, доменные границы начинают совершать колебания с частотой поля относительно своих положений равновесия. При увеличении амплитуды поля $H_{0}$ выше некоторого критического значения $H_{\mathrm{dr}}$ в образце помимо колебаний ДГ возникает трансляционное движение полосовой ДС как целого (дрейф), и в определенном интервале амплитуд поля 
колебания и дрейф ДГ сосуществуют. При дальнейшем возрастании $H_{0}$ наблюдается разрушение полосовой ДС и движение ДГ становится неупорядоченным, что рассматривается нами как прекращение дрейфового движения.

На рис. 2 приведены зависимости скорости дрейфа $V_{\mathrm{dr}}$ от амплитуды $H_{0}$ и частоты $f$ магнитного поля для одноосного образца. Видно, что в исследованном частотном

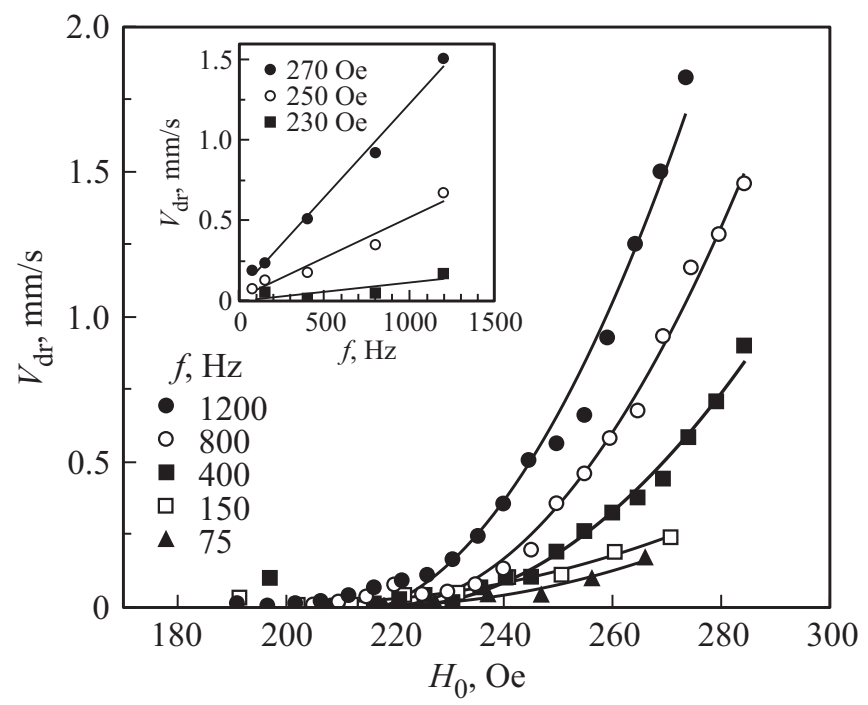

Рис. 2. Зависимости скорости дрейфа $V_{\mathrm{dr}}$ доменных границ одноосного образца от амплитуды гармонического поля $H_{0}$ в диапазоне частот 75-1200 Hz, сплошные линии - аппроксимации экспериментальных зависимостей квадратичными функциями. На вставке - зависимости скорости дрейфа ДГ от частоты поля $f$, построенные по аппроксимациям амплитудных зависимостей.

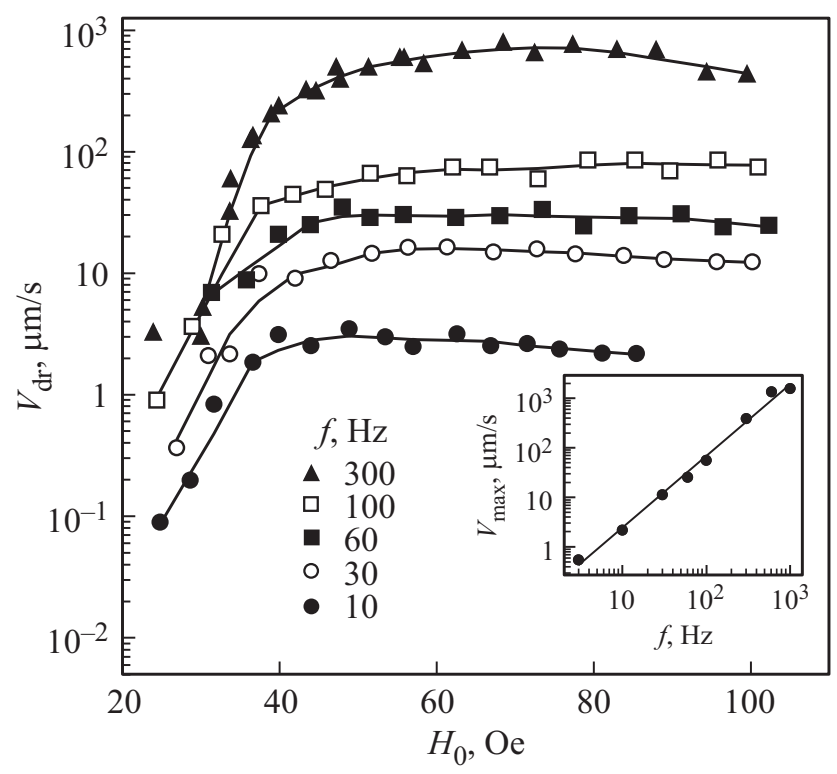

Pис. 3. Зависимости скорости дрейфа $V_{\mathrm{dr}}$ доменных границ многоосного образца от амплитуды поля $H_{0}$ в диапазоне частот $10-300 \mathrm{~Hz}$. На вставке - частотная зависимость максимальной скорости доменных границ $V_{\max }$, достигаемой при фиксированной частоте $f$ магнитного поля.

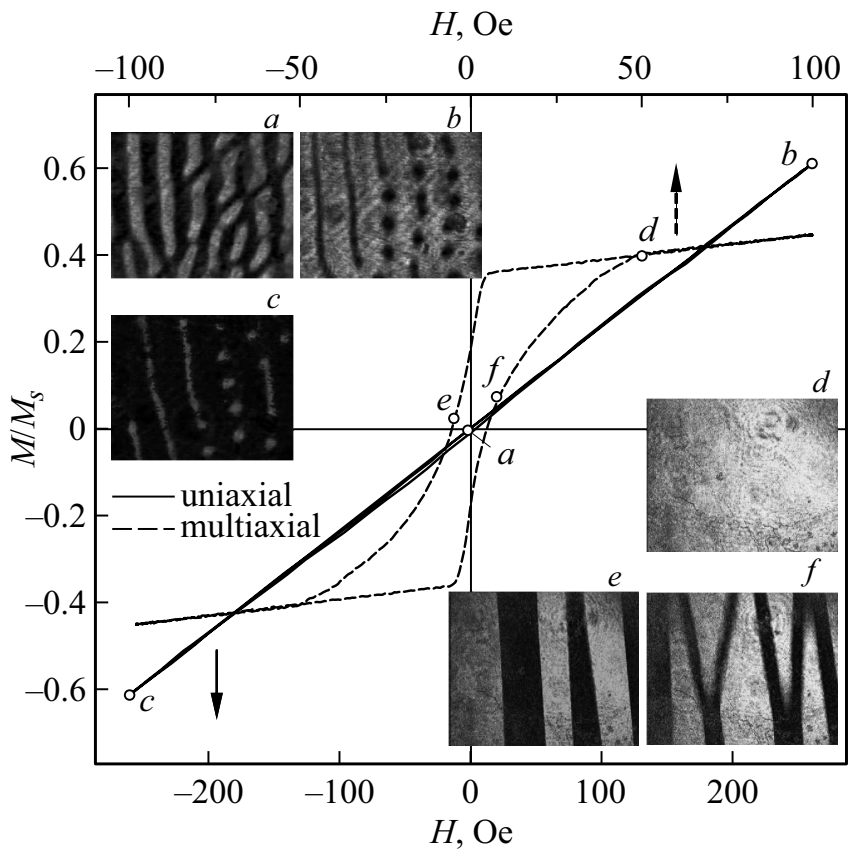

Рис. 4. Частные динамические петли магнитного гистерезиса одноосного (сплошные линии) и многоосного (пунктирные линии) образцов. Петля гистерезиса одноосного образца снята при частоте магнитного поля $f=100 \mathrm{~Hz}$, амплитуде поля $H_{0}=260$ Ое, петля гистерезиса многоосного образца при частоте $f=200 \mathrm{~Hz}$, амплитуде $H_{0}=100$ Ое. На вставках - изображения динамических доменных структур одноосного $(a, b, c)$ и многоосного $(d, e, f)$ образцов. Мгновенные значения напряженности поля $H: a--1, b-260, c--260$, $d-50, e--5, f-10$ Oe.

диапазоне дрейф ДГ происходит при амплитудах поля от 190 до 280 Ое, а скорость дрейфа монотонно возрастает с ростом $H_{0}$. Зависимости $V_{\mathrm{dr}}\left(H_{0}\right)$ аппроксимированы квадратичными функциями (сплошные линии на рис. 2). C увеличением частоты $f$ магнитного поля скорость дрейфа $V_{\mathrm{dr}}$ линейно возрастает (см. вставку на рис. 2).

Зависимость скорости дрейфа от амплитуды поля для многоосного образца приведена на рис. 3. Видно, что она существенно отличается от аналогичной зависимости для одноосного кристалла. На рис. 3 использован логарифмический масштаб, поскольку дрейф при частоте $f=300 \mathrm{~Hz}$ происходит более чем в 200 раз быстрее, чем при минимальной исследованной частоте $f=10 \mathrm{~Hz}$. Пороговые значения амплитуды поля начала дрейфа лежат в области 20-30 Ое. На начальном участке (20-50 Ое) скорость дрейфа ДГ возрастает с ростом амплитуды поля, затем достигает максимума и практически не меняется до $H_{0} \approx 100 \mathrm{Oe}$, когда дрейф прекращается. На вставке к рис. 3 приведена зависимость от частоты максимальной скорости дрейфа ДГ $V_{\max }$, достигаемой при фиксированной частоте поля. В исследованном диапазоне частот эта зависимость имеет линейный характер.

Различный характер зависимости скорости дрейфа от амплитуды переменного поля для образцов с разным типом магнитной анизотропии (одноосной, многоосной) 

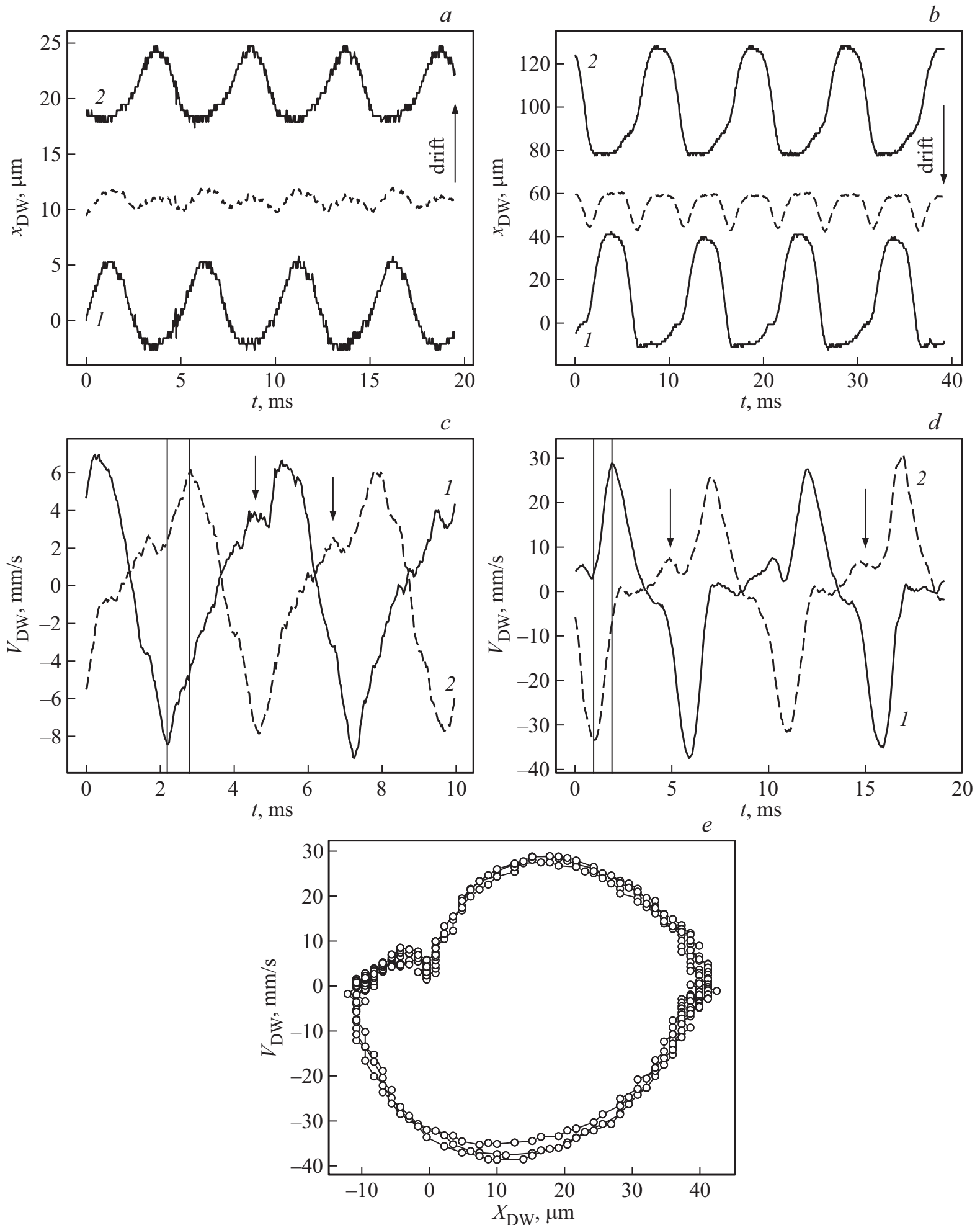

Рис. 5. Зависимости координат соседних доменных границ $x_{\mathrm{DW}}$ (кривые 1,2 ) и центра полосового домена (пунктирные линии) от времени в гармоническом магнитном поле для $a-$ одноосного $\left(f=200 \mathrm{~Hz}, H_{0}=138\right.$ Ое $)$ и $b-$ многоосного образца $\left(f=100 \mathrm{~Hz}, H_{0}=19 \mathrm{Oe}\right) ; c, d-$ соответствующие зависимости от времени мгновенных скоростей $V_{\mathrm{DW}}$ соседних ДГ $(1,2)$ в одноосном $(c)$ и многоосном $(d)$ образцах; $e-$ фазовая траектория движения ДГ в многоосном образце.

коррелирует с качественно отличающимися процессами намагничивания и перемагничивания этих образцов в переменном магнитном поле. На рис. 4 приведены непредельные динамические магнитооптические петли гистерезиса для одноосного (сплошная кривая) и многоосного (пунктирная кривая) образцов. Петли были 
измерены при амплитудах поля, близких к амплитуде прекращения дрейфа. Петля гистерезиса одноосного образца имеет линейный вид, гистерезис при этом практически отсутствует. Вид петли гистерезиса многоосного образца свидетельствует о наличии гистерезиса при динамическом перемагничивании. Для трех значений поля даны изображения динамических ДС, которые были характерны для различных участков динамических петель гистерезиса.

В одноосном образце в нулевом поле наблюдается преимущественно полосовая ДС, границы которой ориентированы в среднем вдоль оси $[\overline{2} 11]$ (рис. $а$ на вставке к рис. 4). В поле $H= \pm 260$ Ое полосовые домены разрываются и формируются цилиндрические магнитные домены (рис. $b, c$ на вставке к рис. 4), которые дрейфуют со скоростью полосовых доменов, сохраняя выстроенность вдоль полос, из которых они образовались. Ранее дрейф ЦМД в ферритах-гранатах в переменном магнитном поле наблюдался в [16]. Именно описанный выше процесс разрыва полосовых доменов ограничивает зависимости $V_{\mathrm{dr}}\left(H_{0}\right)$ полем $H \approx 280 \mathrm{Oe}$.

В многоосном образце в интервале полей $H_{\mathrm{dr}}-40 \mathrm{Oе}$ намагничивание происходит путем колебаний ДГ с одновременным устоявшимся дрейфом полосовых доменов (рис. $4, e, f)$, здесь зависимость $V_{\mathrm{dr}}\left(H_{0}\right)$ имеет линейный вид для исследованных частот (рис. 3). С увеличением амплитуды поля при $H \geq 50$ Ое образец находится часть периода поля в однодоменном состоянии (рис. 4, $d$ ), в котором вектор намагниченности в образце не совпадает с направлением поля, а ориентирован вдоль оси [111]. Время существования полосовых доменов за период поля уменьшается, это приводит к уменьшению средней скорости дрейфа ДГ, что проявляется в появлении плато на зависимости $V_{\mathrm{dr}}\left(H_{0}\right)$ (рис. 3 ).

Для выявления механизмов дрейфа ДГ были исследованы особенности колебательного движения ДГ в гармоническом магнитном поле при амплитудах $H_{0}<H_{\mathrm{dr}}$. Стробоскопическая методика позволила увеличить число фиксируемых положений ДГ за период поля и более детально изучить процессы, протекающие в течение периода поля. На рис. 5 приведены зависимости координат $x_{\mathrm{DW}}$ двух доменных границ полосового домена от времени в гармоническом поле для одноосного $(a)$ и многоосного $(b)$ образцов (стрелками указаны направления дрейфа, возникающего в образцах при $H_{0}>H_{\mathrm{dr}}$ ). Асимметричность колебаний ДГ хорошо видна на графиках. Форма колебаний ДГ очевидно отлична от синусоидальной, длительность нахождения ДГ и характер их движения вблизи положений максимального отклонения от равновесия различны при смещении ДГ в противоположных направлениях. Центры полосовых доменов (пунктирные линии на рис. $5, a, b$ ) совершают колебания с частотой, удвоенной по сравнению с частотой поля накачки.

На рис. 5, $c, d$ приведены графики зависимостей скорости ДГ $V_{\mathrm{DW}}$ от времени для соседних доменных границ одноосного и многоосного образцов соответственно, полученные дифференцированием временных зависимостей координат ДГ с помощью фильтра СавицкогоГолея [17]. Скорости движения ДГ изменяются асинхронно: в то время, когда скорость одной из стенок не меняется со временем или даже несколько уменьшается (отмечено стрелками на рис. $5, c, d$ ), скорость соседней ДГ резко возрастает. Максимальные значения скоростей соседних ДГ (отмечены вертикальными линиями) достигаются не одновременно. Интервал между моментами достижения максимумов составляет около одной десятой периода магнитного поля. Таким образом, рис. 5, $a-d$ показывают, что графики смещений и скоростей соседних ДГ не обладают зеркальной симметрией относительно центра домена в равновесном состоянии и нулевого уровня скоростей соответственно.

На рис. 5, e представлена экспериментальная фазовая траектория движения ДГ для многоосного образца, ее искаженная форма свидетельствует о возникновении высших гармоник в спектре колебаний границы помимо основной частоты внешнего поля.

\section{4. Доменная структура одноосной пластины феррита-граната в периодическом импульсном магнитном поле}

Обзор исследований динамики ДГ в импульсных магнитных полях приведен в [18]. О дрейфе доменов различной конфигурации при приложении к пленке импульсного поля сообщалось в [16]. В задачу нашей работы входило установить влияние колебаний ДГ, возникающих в импульсном поле, на процесс дрейфового движения. Ниже приведены результаты изучения дрейфа полосовой ДС в одноосном образце феррита-граната при приложении повторяющихся импульсов магнитного поля с чередующейся полярностью перпендикулярно плоскости образца. Дрейф ДГ наблюдался в диапазоне амплитуд импульсов поля от 240 до 300 Ое, границы которого смещались при изменении частоты подачи импульсов.

На рис. 6 приведены данные для биполярного импульсного поля с амплитудой $H_{0}=202 \mathrm{Oe}$, меньшей амплитуды начала дрейфа в импульсном поле $H_{\text {dri }}$. Профиль приложенного к образцу импульсного поля $H(t)$ показан на рис. $6, a$. Соответствующая зависимость координаты $x$ одной из ДГ показана на рис. $6, b$. Здесь $x_{1}$ - максимальное смещение ДГ в направлении последующего дрейфа, $x_{2}$ - в противоположном дрейфу направлении. Из сравнения рис. $6, a$ и $b$ видно, что траектории ДГ $x(t)$ повторяют по форме зависимость $H(t)$. Кроме того, имеет место эффект асимметричного смещения доменных границ, заключающийся в том, что смещение ДГ в направлении последующего дрейфа $x_{1}$ больше, чем смещение $x_{2}$ в противоположном дрейфу направлении. Этот эффект не зависит от четности доменных границ: для всех ДГ смещение в направлении 


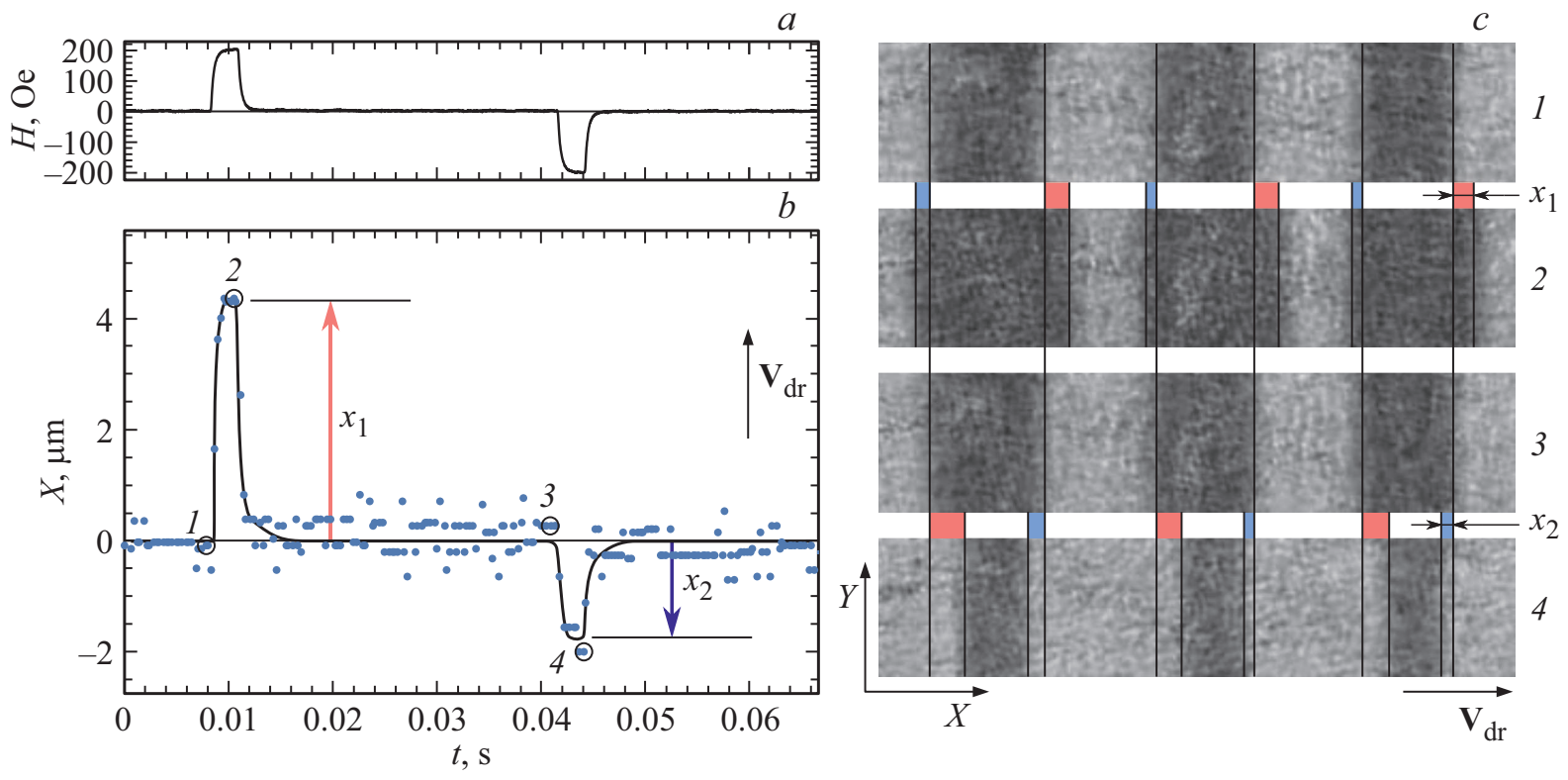

Рис. 6. $a$ - профиль периодического импульсного магнитного поля в течение периода поля, частота подачи импульсов $f=30 \mathrm{~Hz}$, амплитуда $H_{0}=202 \mathrm{Oe}$, длительность импульсов $\tau=2.65 \mathrm{~ms} ; b-$ смещение доменной границы в одноосном образце под действием поля, профиль которого показан на $(a) ; c-$ фотографии динамической ДС одноосного образца, полученные в моменты времени, отмеченные цифрами $1-4$ на рис. $b$, ширина кадров $156 \mu$ m. На рис. $b$ и $c x_{1}-$ величина смещения ДГ в направлении дрейфа при приложении импульса поля по сравнению с состоянием в нулевом поле, $x_{2}$ - величина смещения ДГ в направлении против дрейфа.

дрейфа больше, чем в направлении, противоположном дрейфу.

На рис. 6,c приведены фотографии динамической полосовой ДС при $H=0$ (кадры 1,3 ), на пике положительного импульса поля $H=+H_{0}$ (кадр 2) и на пике отрицательного импульса $H=-H_{0}$ (кадр 4). На кадре 2 видно, что темные домены расширились больше в сторону дрейфа, чем в противоположном дрейфу направлении. Аналогично, на кадре 4 светлые домены расширились больше в направлении дрейфа. После действия импульса поля ДГ возвращаются к своим положениям равновесия (кадры 1,3). Асимметрия в смещении ДГ от положения равновесия показывает, что одно из двух направлений движения ДГ в одноосном образце является предпочтительным. Дрейф в периодическом биполярном импульсном поле происходит именно в этом направлении. Следует отметить, что в одноосном образце в гармоническом поле, приложенном перпендикулярно плоскости образца, дрейф идет в том же направлении, что и в биполярном импульсном поле.

\section{5. Численное моделирование движения ДГ в гармоническом поле}

Численное моделирование движения ДГ в одноосном кристалле проведено в рамках модели вынужденных колебаний системы связанных гармонических осцилляторов [19-22] с параметрами реальных образцов. В рамках данной модели считается, что в процессе движения внутренняя структура ДГ не изменяется, ДГ обладает

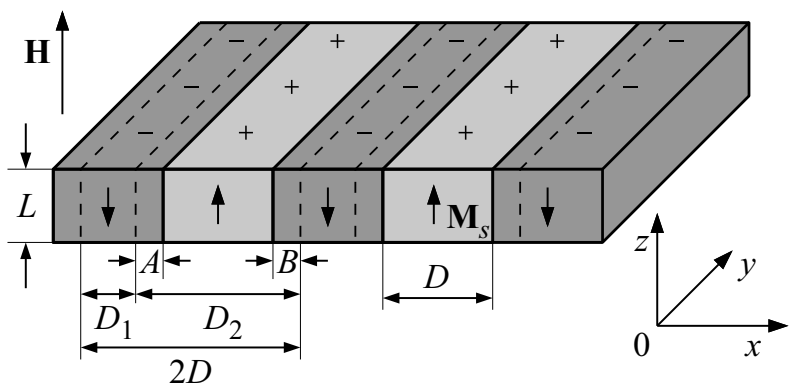

Рис. 7. Модель полосовой доменной структуры.

эффективной массой и движется благодаря действующим на ее поверхность силам давления, которые и являются причиной перемещения ДГ.

На рис. 7 изображена модель полосовой ДС в плоскопараллельной магнитно-одноосной пластине ферритаграната толщиной $L$. Ось легкого намагничивания перпендикулярна плоскости пластины и параллельна оси OZ. Векторы намагниченности $\mathbf{M}_{s}$ в доменах направлены параллельно оси OZ (сонаправлены либо противоположно направлены оси OZ). Ширина полосовых доменов $D$ в отсутствие внешнего магнитного поля определялась экспериментально. Период доменной структуры равен удвоенной ширине домена $2 D$.

Внешнее магнитное поле прикладывается перпендикулярно плоскости образца. В этом случае на ДГ действуют силы: сила со стороны внешнего магнитного поля, приводящая к смещению ДГ из положения равновесия, 
„возвращающая“ сила, связанная с магнитостатической энергией полосовой ДС, и сила динамического трения, связанная с затуханием прецессии намагниченности в магнитном поле. Коэрцитивность ДГ в настоящей работе не учитывается.

С увеличением магнитного поля как вдоль $(H>0)$, так и против $(H<0)$ оси $\mathrm{OZ}$ происходит увеличение ширины доменов, вектор намагниченности в которых сонаправлен с магнитным полем. Смещения доменных границ от положения, занимаемого ими в отсутствии магнитного поля, обозначены как $A$ и $B$ (см. рис. 7). Эти смещения являются одинаковыми для всех периодов ДС. При изменении магнитного поля во времени параметры доменной структуры изменяются, при этом для всех периодов $D_{1}=D-A-B, D_{2}=D+A+B$.

Приложение пространственно однородного осциллирующего магнитного поля в случае однородного изотропного образца приводит к осцилляциям ДГ в модели, но не приводит к дрейфу ДС. В [20] показано, что дрейф при описанных выше условиях может быть вызван неоднородностью магнитного поля или намагниченности в образце.

В настоящей работе в отличие от работ [20-22] предполагается, что в образце, находящемся в пространственно однородном магнитном поле, направленном перпендикулярно плоскости образца, имеется анизотропия параметра затухания: величина параметра затухания различна при движении ДГ в разных направлениях вдоль оси OX, перпендикулярной плоскости доменных границ. Сделать такое предположение позволяет наблюдаемая экспериментально разность смещений ДГ при приложении перпендикулярно плоскости образца импульсов поля различной полярности (см. рис. 6). Учитывая симметрию задачи можно записать уравнения движения для двух соседних ДГ, что является достаточным для описания движения всех ДГ в рассматриваемой модели доменной структуры.

Плотности энергии $\gamma_{H}$ внешнего магнитного поля $H=H_{0} \sin (2 \pi f t)$ и магнитостатической энергии $\gamma_{m}$ имеют следующий вид (см., например, $[23,24])$ :

$$
\begin{gathered}
\gamma_{H}=M_{S} H\left(1-\frac{D_{1}}{D}\right) \sin \omega t, \\
\gamma_{m}=2 \pi M_{S}^{2}\left(\frac{D_{1}-D_{2}}{2 D}\right)^{2}+\frac{16 M_{S}^{2} D}{\pi^{2} L} \\
\times \sum_{n=1}^{\infty}\left\{\frac{1}{n^{3}}\left[1-\exp \left(\frac{-\pi n L}{D}\right)\right] \sin ^{2}\left(\frac{\pi n D_{1}}{2 D}\right)\right\} .
\end{gathered}
$$

Дифференцируя эти выражения по смещениям ДГ А и $B$ и учитывая анизотропию параметра затухания, можем записать уравнения движения для двух соседних ДГ. В безразмерных переменных уравнения движения ДГ будут иметь следующий вид:

$$
\left\{\begin{array}{l}
\frac{\partial^{2} a}{\partial \tau^{2}}+\eta\left(\operatorname{sgn}\left(\frac{\partial a}{\partial \tau}\right)\right) \frac{\partial a}{\partial \tau}+g(a, b)-\pi h_{0} \sin (\nu \tau)=0, \\
\frac{\partial^{2} b}{\partial \tau^{2}}+\eta\left(-\operatorname{sgn}\left(\frac{\partial b}{\partial \tau}\right)\right) \frac{\partial b}{\partial \tau}+g(a, b)-\pi h_{0} \sin (\nu \tau)=0 .
\end{array}\right.
$$

Здесь $a=2 \pi A / D, b=2 \pi B / D-$ безразмерные переменные, соответствующие смещениям левой и правой доменных стенок, $\ell=\pi L / D, h_{0}=H_{0} / 4 \pi M_{s}, \omega=2 \pi f$, $v=\omega / \Omega, \tau=t \Omega, \Omega^{2}=8 \pi M_{s}^{2} / m D, m-$ эффективная масса ДГ,

$$
\begin{aligned}
g(a, b) & =\frac{a+b}{2} \\
+ & \frac{2}{\ell} \sum_{n=1}^{\infty}\left[\frac{(-1)^{n}}{n^{2}}[1-\exp (-n \ell)] \sin \frac{n(a+b)}{2}\right] .
\end{aligned}
$$

Функция затухания $\eta(q)$ определяется следующим образом:

$$
\eta(q)=\left\{\begin{array}{ll}
\eta_{1}, & \text { при } q>0 \\
\eta_{2}, & \text { при } q<0
\end{array},\right.
$$

где $\eta_{i}=\alpha_{i}\left(D /\left(\tilde{A} / K_{u}\right)^{1 / 2}\right)^{1 / 2}-$ нормированные параметры затухания, $\alpha_{i}$ - параметры затухания Гильберта, типичные для рассматриваемых материалов $(i=1,2)$, $\tilde{A} \approx 10^{-7} \mathrm{erg} / \mathrm{cm}$ - параметр обменного взаимодействия.

Система уравнений решалась в пакете Wolfram Mathematica (C) (LSODA Solver [25]) с однородными начальными условиями.

Численное моделирование показывает, что в предположении различия величины параметра затухания при движении ДГ в разных направлениях вдоль оси, перпендикулярной плоскости доменных границ, в движении соседних ДГ под действием гармонических магнитных полей имеется асимметрия, приводящая к дрейфу ДГ. При этом центры доменов колеблются с частотой, равной удвоенной частоте внешнего магнитного поля, что согласуется с экспериментальными данными.

Скорость дрейфа ДС изменяется при изменении отношения параметров затухания $\eta_{1} / \eta_{2}$. Дрейф со скоростями, наблюдаемыми экспериментально, получается в модели при отношении параметров затухания порядка 1.09. На рис. 8, $a-c$ показаны зависимости координат ДГ (кривые 1 и 2) и координат центра домена от времени при отношении параметров затухания $\eta_{1} / \eta_{2}=4$ с целью проиллюстрировать колебания центра домена и асимметрию движения границ, которые менее выражены при меньших отношениях параметров затухания. На рис. $8, c, d$ видно, что движение ДГ в модели в направлении дрейфа происходит с большей скоростью, чем в противоположном дрейфу направлении, а максимальные отклонения ДГ от положения равновесия достигаются не синхронно - всякий раз доменная граница, которая движется в направлении дрейфа, достигает максимального отклонения от положения равновесия раньше границы, которая движется в противоположном дрейфу направлении.

В отличие от [20], предположение об анизотропии параметра затухания в плоскости образца позволяет получить зависимости скорости дрейфа $V_{\mathrm{dr}}$ от амплитуды и частоты внешнего гармонического магнитного поля, являющиеся, как и экспериментально полученные 

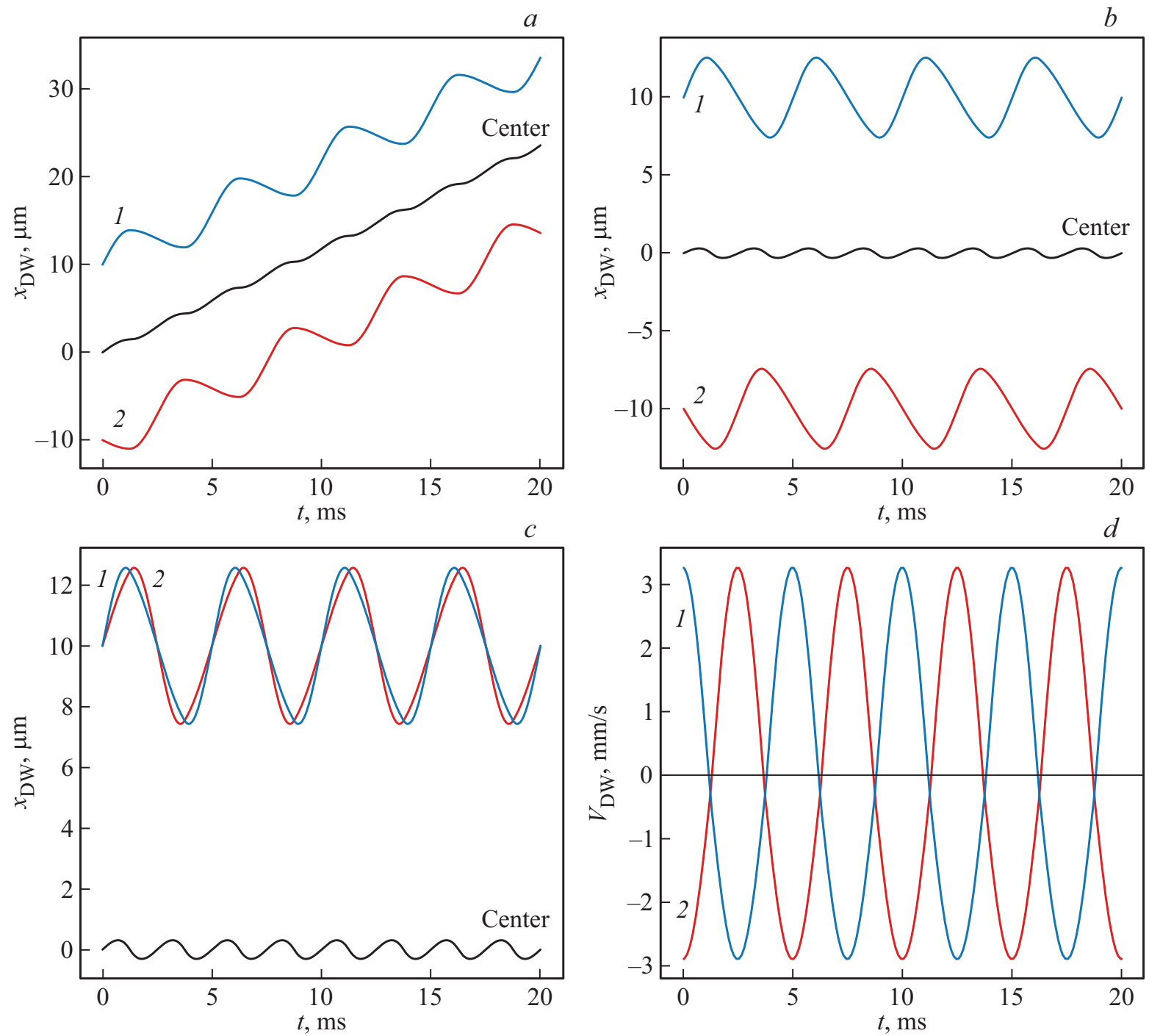

Рис. 8. Полученные в результате численного моделирования зависимости координат ДГ (кривые 1,2$)$ и координат центра домена от времени при $\eta_{1} / \eta_{2}=4, H=138 \mathrm{Oe}, f=200 \mathrm{~Hz}: a-$ при наличии дрейфа; $b-$ смещение, соответствующее дрейфу, вычтено из смещений ДГ; $c$ - колебания соседних ДГ наложены друг на друга. $d-$ зависимости от времени скорости $V_{\mathrm{DW}}$ соседних ДГ при $\eta_{1} / \eta_{2}=1.4, H=138$ Ое и $f=200 \mathrm{~Hz}$.

зависимости, возрастающими функциями $H_{0}$ и $f$ (рис. 9). Линейный характер зависимостей $V_{\mathrm{dr}}(f)$ согласуется с результатами эксперимента (см. вставку на рис. 2). В зависимостях $V_{\mathrm{dr}}\left(H_{0}\right)$ присутствует слабая нелинейность.

\section{6. Обсуждение результатов}

Наши наблюдения и анализ литературных данных позволяют считать, что дрейф ДС является этапом динамического перемагничивания в переменном магнитном поле. В исследованных пластинах с разным типом магнитной анизотропии полученные зависимости скорости дрейфа ДГ от амплитуды гармонического поля качественно различны, что коррелирует с различным характером намагничивания и перемагничивания одноосного и многоосного образцов. Динамическая пере- стройка ДС в поле определяет диапазон дрейфа ДГ, а также появление плато на амплитудной зависимости скорости дрейфа для многоосного образца (см. рис. 3).

Если для кубических кристаллов теория дрейфа ДГ не построена, то для одноосных образцов теория дрейфа в линейно поляризованном гармоническом магнитном поле рассмотрена в ряде работ [7-9]. В [8] построена феноменологическая теория дрейфа ДГ в ферромагнетиках, обладающих кубической магнитокристаллической анизотропией с отрицательной константой $K_{1}<0$ и наведенной одноосной анизотропией. Это соответствует свойствам исследованных в нашей работе кристаллов ферритов-гранатов. В [8] получена квадратичная зависимость скорости дрейфа от амплитуды поля, что согласуется с полученными нами экспериментальными зависимостями для одноосного образца. При этом, согласно [8], при частотах поля, много меньших частоты 

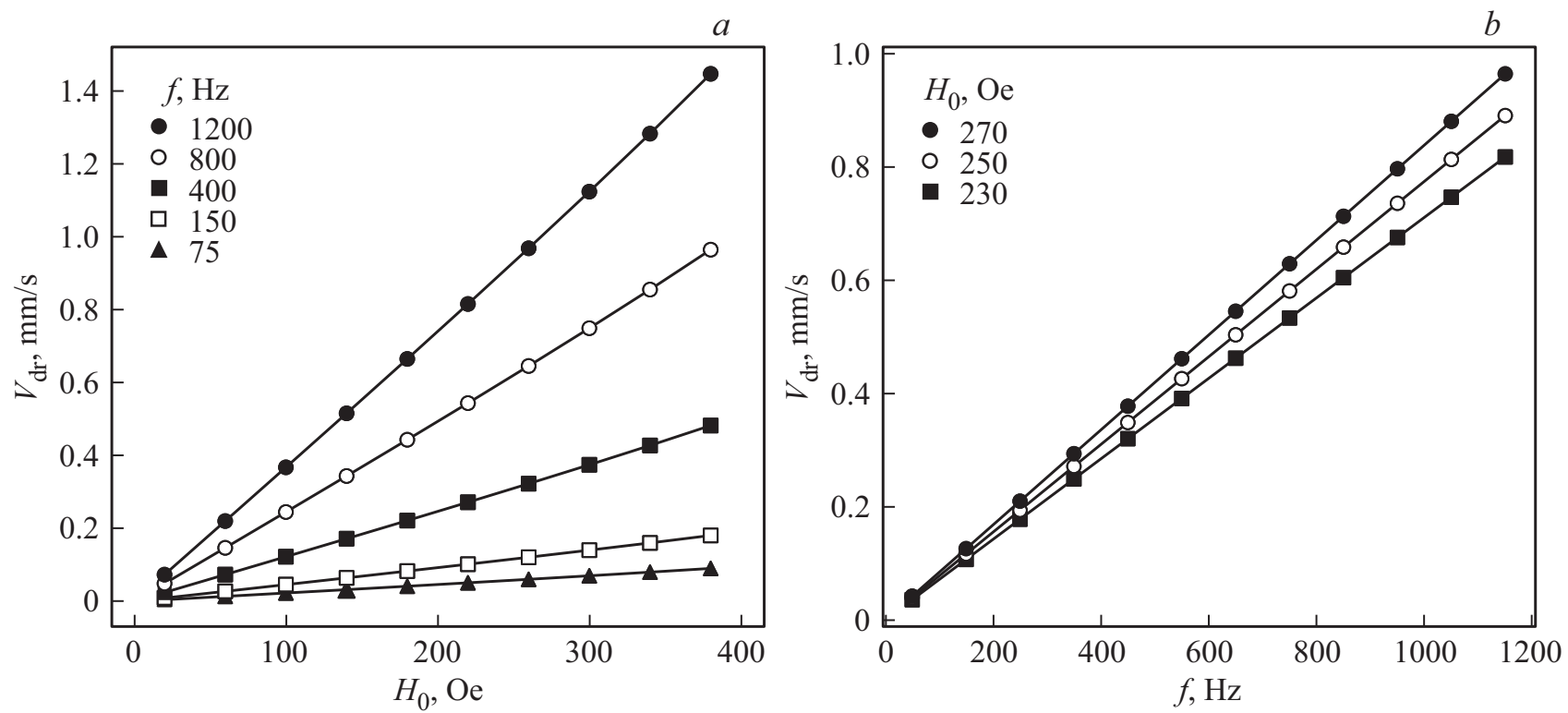

Рис. 9. Полученные в результате численного моделирования зависимости скорости дрейфа доменных границ $V_{\mathrm{dr}}$ от $a-$ амплитуды $H_{0}$ и $b-$ частоты $f$ внешнего гармонического магнитного поля при $\eta_{1} / \eta_{2}=1.09$.

однородного ферромагнитного резонанса, зависимость скорости дрейфа от частоты поля отсутствует, тогда как результаты наших экспериментов свидетельствуют о линейной зависимости скорости дрейфа от частоты как для одноосного, так и для многоосного образца.

В образцах с разным типом магнитной анизотропии экспериментально установлены общие особенности колебаний ДГ в гармоническом магнитном поле: a) наличие асимметрии в смещении ДГ (рис. 5, $a, b$ ); б) асинхронный характер в изменении скоростей соседних ДГ в течение периода поля (рис. 5,c,d); в) удвоенная частота колебаний центров доменов по сравнению с частотой внешнего поля.

Исследования движения ДГ в одноосном образце в импульсном поле показали, что смещение ДГ в направлении дрейфа существенно превосходит смещение этой же границы в противоположном дрейфу направлении. Это позволяет предположить, что направление дрейфа ДГ связано с направлением максимального смещения ДГ в импульсном магнитном поле с амплитудой $H_{0}<H_{\text {dri }}$. Наблюдающееся различие в величине смещений, а следовательно, и скоростей движения доменных границ, может быть связано с разными значениями параметра затухания при движении ДГ в направлении наблюдаемого дрейфа и в противоположном дрейфу направлении.

Предположение об анизотропии параметра затухания использовано нами в численном моделировании колебаний и дрейфа ДГ, проведенном в рамках модели связанных осцилляторов с параметрами исследованных образцов. О наличии анизотропии параметра затухания Гильберта сообщалось в [26-28]. Сделанное в модели предположение о наличии в материале анизотропии параметра затухания дает возможность получить зависимости скорости дрейфа от амплитуды и частоты внешнего магнитного поля (рис. 9), являющиеся, как и экспериментально полученные зависимости, возрастающими функциями амплитуды и частоты. Зависимость $V_{\mathrm{dr}}$ от $f$ является линейной согласно экспериментальным данным и результатам численного моделирования (рис. 2 и рис. $9, b)$. Численное моделирование показывает, что смещения соседних ДГ асимметричны (рис. 8, b, $c$ ), а центры доменов совершают колебания с удвоенной частотой поля накачки (рис. $8, a-c$ ). Экспериментально наблюдаемая картина колебаний ДГ более сложна, однако используемая теоретическая модель достаточно адекватно отражает основные закономерности колебательного и дрейфового движения ДГ в гармоническом поле.

\section{7. Заключение}

Обнаружена асимметрия смещения доменных границ в гармоническом и периодически повторяющемся импульсном магнитных полях в пластинах ферритовгранатов с разным типом преобладающей магнитной анизотропии.

Установлено, что при амплитудах биполярного импульсного магнитного поля, меньших амплитуды поля начала дрейфа в одноосном образце, смещение ДГ в направлении последующего дрейфа больше смещения этой же ДГ в противоположном дрейфу направлении. Это позволяет предполагать, что направление дрейфа определяется характером смещений доменных границ в процессе колебаний.

Численное моделирование движения ДГ в гармоническом магнитном поле показывает, что асимметрия смещения доменных границ может быть связана с наличием анизотропии параметра затухания в плоскости образца. Полученные в модели зависимости скорости дрейфа 
доменных границ от амплитуды и частоты внешнего гармонического поля согласуются с установленными экспериментально.

\section{Список литературы}

[1] S.S.P. Parkin, M. Hayashi, L. Thomas. Science 320, 5873, 190 (2008).

[2] J.H. Franken, H.J.M. Swagten, B. Koopmans. Nature Nanotechnology 7, 8, 499 (2012).

[3] K.W. Moon, D.H. Kim, S.C. Yoo, S.G. Je, B.S. Chun, W. Kim, B.C. Min, C. Hwang, S.B. Choe. Sci. Rep. 5, 9166 (2015).

[4] B. Passon. Z. Angew. Phys. 25, 2, 56 (1968).

[5] В.К. Власко-Власов, Л.С. Успенская. ЖЭТФ 91, 4, 1483 (1986).

[6] А.Б. Чижик, С.Л. Гнатченко. ФНТ 21, 5, 538 (1995).

[7] В.Г. Барьяхтар, Ю.И. Горобец, С.И. Денисов. ЖЭТФ 98, 4, 1345 (1990).

[8] Г.Е. Ходенков. Письма в ЖЭТФ 68, 11, 823 (1998).

[9] Y. Zolotaryuk, M.M. Osmanov. Eur. Phys. J. B 79, 3, 257 (2011).

[10] E. Schlömann, J.D. Milne. IEEE Trans. Magn. 10, 791 (1974).

[11] В.С. Герасимчук, А.Л. Сукстанский. ЖЭТФ 103, 1, 151 (1993).

[12] М.М. Соловьёв, Б.Н. Филиппов. ФММ 98, 3, 12 (2004).

[13] Ю.Н. Драгошанский, Е.Б. Хан, В.А. Зайкова. ФММ 39, 2, 289 (1975).

[14] Л.А. Памятных, М.С. Лысов, Г.С. Кандаурова. Изв. РАН. Сер. физ. 71, 11, 1542 (2007).

[15] L.A. Pamyatnykh, B.N. Filippov, L.Y. Agafonov, M.S. Lysov. Sci. Rep. 7, 1, 18084 (2017).

[16] Ф.В. Лисовский, Е.Г. Мансветова, Ч.М. Пак. ЖЭТФ 108, 3, 1031 (1995).

[17] A. Savitzky, M.J.E. Golay. Anal. Chem. 36, 8, 1627 (1964).

[18] В.В. Волков, В.А. Боков. ФТТ 50, 2, 193 (2008).

[19] S. Chikazumi. Physics of ferromagnetism. 2nd ed. Oxford University Press, N.Y. (2009). 682 p.

[20] L.A. Pamyatnykh, G.A. Shmatov, S.E. Pamyatnykh, M.S. Lysov, D.S. Mekhonoshin, A.V. Druzhinin. Acta Phys. Polonica A 127, 2, 388 (2015).

[21] L.A. Pamyatnykh, L.Y. Agafonov, D.S. Mekhonoshin, S.E. Pamyatnykh, M.S. Lysov, G.A. Shmatov. Phys. Procedia 75, 986 (2015)

[22] L.A. Pamyatnykh, G.A. Shmatov, M.S. Lysov, S.E. Pamyatnykh, D.S. Mehonoshin. Solid State Phenomena 215, 437 (2014).

[23] C. Kittel. Rev. Mod. Phys. 21, 4, 541 (1949).

[24] Г.С. Кандаурова, Л.Г. Оноприенко. Основные вопросы теории магнитной доменной структуры. УрГУ, Свердловск (1977). $124 \mathrm{c}$.

[25] A. Hindmarsh, R.S.S.E. Al. IMACS Trans. Sci. Comp. 1, 55 (1983).

[26] В.В. Рандошкин, Ю.Н. Сажин. ЖТФ 66, 8, 83 (1996).

[27] А.В. Кобелев, Ю.Н. Швачко, В.В. Устинов. ФММ 117, 1, 11 (2016).

[28] K. Gilmore, M.D. Stiles. Phys. Rev. B 81, 17, 174414 (2010).

Редактор Т.Н. Василевская 